\title{
Investigations on structural disorder-induced modifications in the transport behaviour of rare-earth manganites
}

\author{
ZALAK JOSHI $^{1}$, D D PANDYA ${ }^{1}$, DAVIT DHRUV ${ }^{1,2}$, KEVAL GADANI ${ }^{1}$, HETAL BORICHA ${ }^{1}$, \\ SANJAY KANSARA ${ }^{1}$, J H MARKNA ${ }^{2}$, P S SOLANKI ${ }^{1}$ and N A SHAH ${ }^{1, *}$ \\ ${ }^{1}$ Department of Physics, Saurashtra University, Rajkot 360 005, India \\ ${ }^{2}$ V.V.P. Engineering College, Gujarat Technological University, Rajkot 360 005, India
}

MS received 7 December 2015; accepted 2 February 2016

\begin{abstract}
The results of the studies on structural disorder-induced modifications in the transport behaviour of $\mathrm{La}_{0.5} \operatorname{Pr}_{0.2} \mathrm{Ca}_{0.3-x} \mathrm{Ba}_{x} \mathrm{MnO}_{3}$ (LPCBMO) $(0.05 \leq x \leq 0.30)$ manganites were reported. Structural studies using $\mathrm{X}$-ray diffraction (XRD) measurements confirmed the single phasic nature of all the samples without any detectable impurities. The A-site size disorder $\left(\sigma_{\mathrm{A}}^{2}\right)$ increased from $3.81 \times 10^{-5}(x=0.05)$ to $14.9 \times 10^{-5}(x=0.30)$. With the increase in structural disorder in LPCBMO system, the transport improved for the range: $0.15 \leq x \leq 0.30$, which can be ascribed to the enhancement in one electron bandwidth which dominates over the structural disorder effect, while for lower values of $x$, strong competition existed between size disorder and one electron bandwidth. Below $50 \mathrm{~K}$, all $\rho-T$ plots showed resistivity minimum behaviour, which modified with disorder. This behaviour was discussed in detail on the basis of electron-electron interaction having the form: $\rho=\left[1 /\left(\sigma_{0}+B T^{1 / 2}\right)\right]+\rho_{n} T^{n}$. Variation in temperature sensitivity with disorder was also discussed in context of granular morphology and phase segregation scenario.
\end{abstract}

Keywords. Manganites; structural disorder; transport behaviour; phase segregation.

\section{Introduction}

Discovery of colossal magnetoresistance (CMR) in hole-doped mixed valent $\mathrm{ABO}_{3}$ type perovskite manganites, $\mathrm{R}_{1-x} \mathrm{~A}_{x} \mathrm{MnO}_{3}$ (where $\mathrm{R}$ is the rare-earth element and $\mathrm{A}$ the divalent ion), has triggered considerable scientific and technological interest owing to their remarkable electrical and magnetic properties [1-3]. Manganites are known to exhibit some interesting structural and physical properties such as metal-insulator transition $\left(T_{\mathrm{P}}\right)$ in concomitant with ferromagnetic-paramagnetic transition $\left(T_{\mathrm{C}}\right)$, large magnetoresistance $(\mathrm{MR})$ at $\sim T_{\mathrm{P}}$ and at low temperature $<100 \mathrm{~K}$, spin polarization, charge ordering $(\mathrm{CO})$, orbital ordering $(\mathrm{OO})$, spin ordering (SO), size disorder effect, etc. By engineering various parameters of manganites, one can grow thin films and devices consisting of manganites for various practical applications such as spintronic applications, sensors, spin valves, magnetic tunnel junctions, $\mathrm{p}-\mathrm{n}$ junctions, field effect devices, etc. [4-8].

Since, practical applications of manganites demand thin film and device forms of the material, it is also important to understand the intrinsic behaviour of manganites and their physical properties in polycrystalline bulk form wherein no strain (generated due to lattice mismatch between the film and substrate) exists and no control over the degrees of freedom. Conductivity and ferromagnetism in manganites can

\footnotetext{
*Author for correspondence (snikesh@yahoo.com)
}

be explained on the basis of Zener double exchange (ZDE) mechanism $[9,10]$, while structural disorder related to $\mathrm{MnO}_{6}$ octahedral can be explained by Jahn-Teller (JT) distortion $[11,12]$. This was traditionally understood within the framework of ZDE between $\mathrm{Mn}^{3+}$ and $\mathrm{Mn}^{4+}$ via $\mathrm{O}^{2-}$ in which the motion of $e_{\mathrm{g}}$ electron between the two partially filled d shells with strong Hund's coupling results in the magnetic coupling between $\mathrm{Mn}^{3+}$ and $\mathrm{Mn}^{4+}$ ions [9,10]. Later, it was realized that ZDE mechanism alone cannot explain the transport in manganites [11]. It is suggested that a strong electronphonon coupling due to JT effect of $\mathrm{Mn}^{3+}$ ion should play a key role in the localization of charge carriers and the origin of CMR effect [12].

Mainly, transport properties in manganites can be controlled by the following parameters such as size variance, tolerance factor, transfer integral and carrier density are discussed as follows:

(i) Goldschmidt tolerance factor [13] defined by

$$
t=\left(r_{\mathrm{A}}+r_{\mathrm{O}}\right) /\left[\sqrt{ } 2 \times\left(r_{\mathrm{B}}+r_{\mathrm{O}}\right)\right],
$$

where $r_{\mathrm{A}}, r_{\mathrm{B}}$ and $r_{\mathrm{O}}$, respectively, are the average Asite, $\mathrm{B}$-site cationic radii and oxygen ionic radius.

(ii) Charge carrier density determined by the amount of hole doping ( $x$ in $\mathrm{R}_{1-x} \mathrm{~A}_{x} \mathrm{MnO}_{3}$ ).

(iii) Size variance calculated using the formula

$$
\sigma^{2}\left(r_{\mathrm{A}} \text { or } r_{\mathrm{B}}\right)=\left\langle r_{\mathrm{A} / \mathrm{B}}^{2}\right\rangle-\left\langle r_{\mathrm{A} / \mathrm{B}}\right\rangle^{2}=\sum x_{\mathrm{i}} r_{\mathrm{i}}^{2}-\left(\sum x_{\mathrm{i}} r_{\mathrm{i}}\right)^{2},
$$


which depends on an average A-site (or the B-site) cationic radius ( $x_{\mathrm{i}}$ is a fractional occupancy and $r_{\mathrm{i}}$ the corresponding ionic radius).

As discussed above, the tolerance factor and size variance are the calculated parameters, while charge carrier density directly affects the transfer integral as well as other physical properties of the mixed valent manganites. In addition, size of the dopant ( $\mathrm{A}$ in $\mathrm{R}_{1-x} \mathrm{~A}_{x} \mathrm{MnO}_{3}$ ) decides the one electron bandwidth in the system. Few reports are available on the studies of structural disorder-induced modifications in the transport, magnetotransport and magnetic properties of mixed valent manganites [14-18]. Rana et al [19] have studied the low temperature transport properties of $\mathrm{La}_{0.5} \mathrm{Pr}_{0.2} \mathrm{Ba}_{0.3} \mathrm{MnO}_{3}$ (LPBMO) $(x=0.30)$ manganite compound and observed structural disorder-induced resistivity minima at low temperature $\sim 45 \mathrm{~K}$. In recent times, Solanki et al [20] have investigated the thickness-dependent resistivity minimum behaviour in $\mathrm{La}_{0.7} \mathrm{~Pb}_{0.3} \mathrm{MnO}_{3}$ films. Also, reports are available on the manganite-based thin film devices exhibiting large temperature and field sensitivity which are important for their practical applications [6,7].

Several reports exist on the transport and magnetoresistance studies on $\mathrm{La}_{1-x} \mathrm{Ca}_{x} \mathrm{MnO}_{3}$ (LCMO) and $\mathrm{Pr}_{1-x} \mathrm{Ca}_{x}$ $\mathrm{MnO}_{3}$ (PCMO) mixed valent manganites. LCMO exhibits prominent electrical and magnetic transitions for $x=0.1-0.5$, while for $x>0.5$, it shows predominant charge ordering behaviour [21]. Optimum transitions have been observed for $x=0.3$. Also, it shows large MR in the vicinity of transition temperatures. PCMO exhibits complete distinct behaviour for various substitution levels of $\mathrm{Ca}$ [22]. This system does not show any ferromagnetism with high conductivity. Insulating nature of PCMO system is mainly due to the smaller $\mathrm{Pr}^{3+}$ size as compared to $\mathrm{La}^{3+}$. Smaller size of $\mathrm{Pr}^{3+}$ reduces the superexchange competitive with ZDE [23]. Although there is no any electrical transition, the system exhibits ferromagnetism across the range of $0.2 \leq x \geq 0.3$ [24]. Existence of ferromagnetism can be ascribed to the high magnetic moment of $\mathrm{Pr}^{3+}$ ion. On the other hand, $\mathrm{Ba}^{2+}$ (radius $\sim 1.47 \AA$ ) is the large size cation which induces an effective size disorder and structural distortions in the manganite system. These observations encouraged us to study the simultaneous effect of $\mathrm{Pr}^{3+}$ and $\mathrm{Ba}^{2+}$ in $\mathrm{La}_{07} \mathrm{Ca}_{0.3} \mathrm{MnO}_{3}$ system.

Considering all the above studies and aspects of manganites, in the present paper, the results of the studies on Ba-substitution-dependent resistivity behaviour and lowtemperature resistivity minimum behaviour in $\mathrm{La}_{0.5} \operatorname{Pr}_{0.2}$ $\mathrm{Ca}_{0.3-x} \mathrm{Ba}_{x} \mathrm{MnO}_{3}$ (LPCBMO) $(0.05 \leq x \leq 0.30)$ manganites were reported and the results have been discussed in the light of Ba-content-induced modifications in structural disorder, one electron bandwidth and electron-electron (e-e) interactions. The addition of larger $\mathrm{Ba}^{2+}(\sim 1.47 \AA)$ at smaller $\mathrm{Ca}^{2+}$ $(\sim 1.18 \AA)$ site in LPCBMO $(0.05 \leq x \leq 0.30)$ manganites enhances the structural disorder in the system. Large temperature sensitivity has been observed in the present study of LPCBMO system. In this study, the results of the studies on LPCBMO compounds have been compared with the results of $\mathrm{La}_{0.5} \mathrm{Pr}_{0.2} \mathrm{Ca}_{0.3} \mathrm{MnO}_{3}$ (LPCMO) ( $x=0.00$; starting member of the series) compound, reported earlier by Thaker et al [25] and Mavani et al [26].

\section{Experimental}

LPCBMO $(0.05 \leq x \leq 0.30)$ compounds were synthesized by employing conventional solid state reaction route. Constituent oxides $\left(\mathrm{La}_{2} \mathrm{O}_{3}, \operatorname{Pr}_{6} \mathrm{O}_{11}\right.$ and $\left.\mathrm{MnO}_{2}\right)$ and carbonates $\left(\mathrm{BaCO}_{3}\right.$ and $\mathrm{CaCO}_{3}$ ) were mixed thoroughly in stoichiometric proportions and calcined at $950^{\circ} \mathrm{C}$ for $24 \mathrm{~h}$. These were ground, pelletized and sintered in the temperature range of $1100-1200^{\circ} \mathrm{C}$ for $100 \mathrm{~h}$ with few intermediate grindings. The final sintering was carried out at $1300^{\circ} \mathrm{C}$ for $24 \mathrm{~h}$ in air atmosphere. X-ray diffraction (XRD) patterns were recorded in the $2 \theta$ range of $20-80^{\circ}$ on a Siemens diffractometer. The resistivity and MR measurements were carried using the d.c. four probe method using a physical property measurement system (PPMS, Quantum Design).

\section{Results and discussion}

Figure 1 shows the XRD patterns of all the samples studied. It can be seen that all the samples possess single phasic nature without any detectable impurities within the measurement range of $20-80^{\circ}$. Figure 2 shows the typical Rietveld refined XRD pattern of LPCBMO $(x=0.20)$ sample which confirms the single phasic nature of the samples having distorted orthorhombic structure (space group: Pnma (no. 62)). The similar distorted orthorhombic structure was reported for LPCMO $(x=0.00)$ compound $[25,26]$. Samples possess orthorhombic unit cell, where with the increase in Bacontent $(x)$, lattice parameters and cell volume increase. This can be ascribed to the larger sized $\mathrm{Ba}^{2+}(1.47 \AA \hat{)})$ doped at smaller sized $\mathrm{Ca}^{2+}(1.18$ Á) site. As discussed above, the size

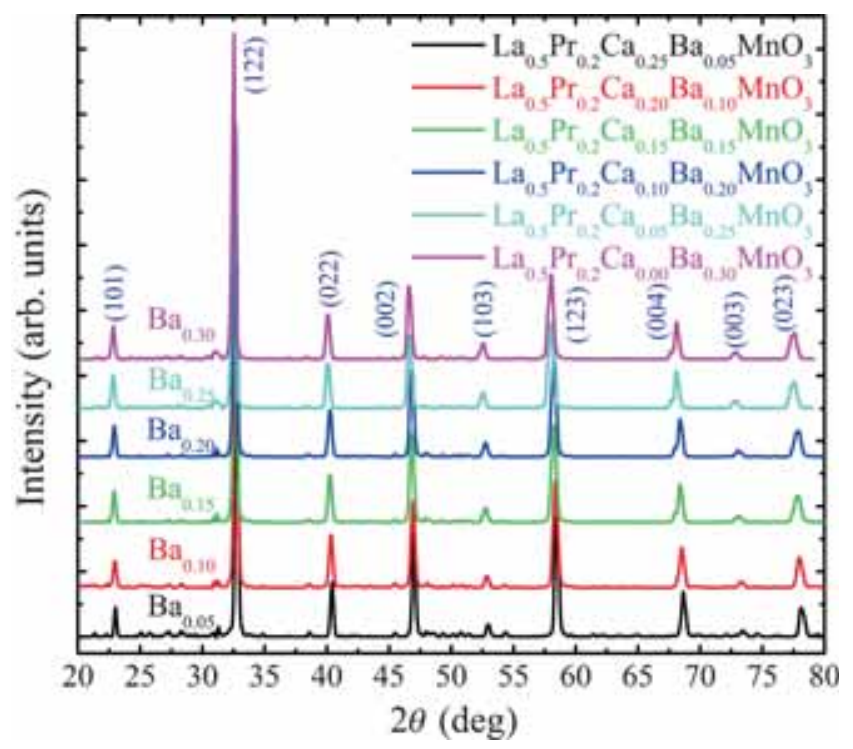

Figure 1. (Colour online) XRD patterns of $\mathrm{La}_{0.5} \operatorname{Pr}_{0.2} \mathrm{Ca}_{0.3-x}$ $\mathrm{Ba}_{x} \mathrm{MnO}_{3}($ LPCBMO; $0.05 \leq x \leq 0.30)$ manganites. 
disorder as one of the effective parameters increases with the increase in Ba-content. Table 1 lists the values of Rietveld refined lattice parameters and cell volume for the present studies of LPCBMO manganites.

To understand the transport behaviour of the present study of LPCBMO manganites, temperature-dependent resistance has been studied in the temperature range of 5-330 K under various applied fields. Figure 3 shows the temperaturedependent variation in resistance of all the LPCBMO samples under 0,5 and $9 \mathrm{~T}$ fields. It is clearly seen that with the increase in applied magnetic field, resistance gets suppressed indicating the negative MR in all the samples understudy. Thaker et al [25] have reported the negative MR in LPCMO sample. Also, with increase in Ba-content, resistance decreases which is more clearly shown in last plot of figure 3 . This can be attributed to the increase in one electron bandwidth with Ba-content, which supports the transfer integral of $e_{\mathrm{g}}$ itinerant electrons and hence enhances the conductivity. It is worth to note that for the samples having lower Ba-content, i.e., $x=0.05$ and 0.10 , value of peak resistance is large in $x=0.05$, while for low and high temperature regions, resistance is observed to be higher in $x=0.10$ sample. This can be ascribed to the competition between the effects of one electron bandwidth (which is large for $x=0.10$ ) and structural disorder (which is small for $x=0.05$ ).

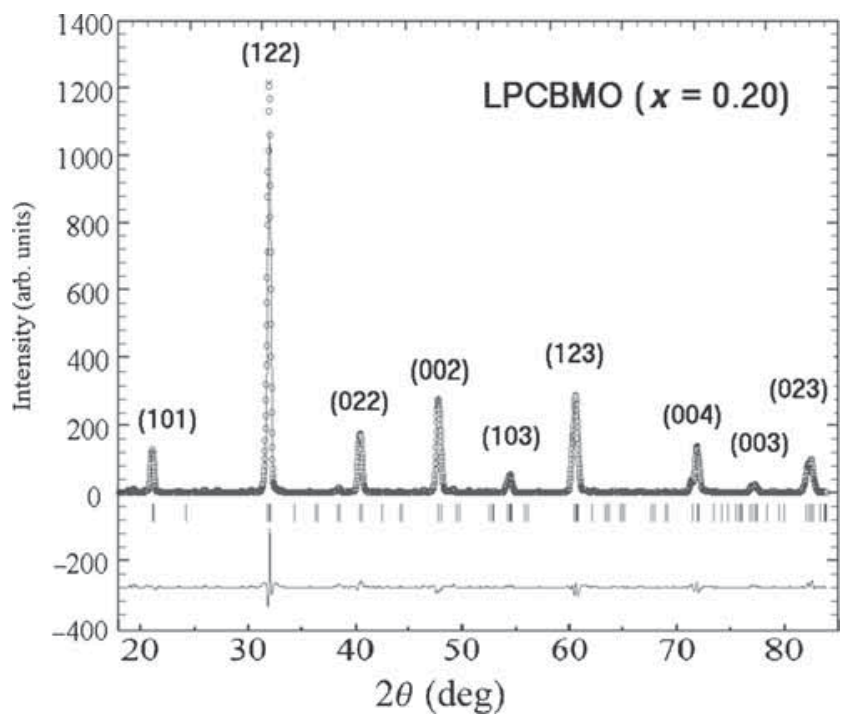

Figure 2. Typical Rietveld refined XRD pattern of $\mathrm{La}_{0.5} \mathrm{Pr}_{0.2}$ $\mathrm{Ca}_{0.3-x} \mathrm{Ba}_{x} \mathrm{MnO}_{3}$ (LPCBMO; $\left.x=0.20\right)$ manganite.
All the samples exhibit metal to insulator transition at $T_{\mathrm{P}}$, which increases with Ba-content (as indicated in the small brackets in figure 3). Thaker et al [25] have reported the transition temperature $\sim 135 \mathrm{~K}$ for LPCMO sample, which increases upon the substitution of $\mathrm{Ba}^{2+}$ at $\mathrm{Ca}^{2+}$ site, as observed for the present case of LPCBMO system. Also, with applied magnetic field, $T_{\mathrm{P}}$ shifts towards higher temperature. Field-induced shift in $T_{\mathrm{P}}$ can be attributed to the fieldinduced suppression in magnetic disorder at $\mathrm{Mn}-\mathrm{O}-\mathrm{Mn}$ bond angles and decrease in scattering of the charge carriers at the grain boundaries. Ba-content induced increase in $T_{\mathrm{P}}$ is due to the improved one electron bandwidth which in turn results into the large transfer integral (which is mainly depends on the $\mathrm{Mn}-\mathrm{O}-\mathrm{Mn}$ bond angles) and hence, ZDE interaction gets improved resulting in the increase in $T_{\mathrm{P}}$ with $\mathrm{Ba}$-content from $147 \mathrm{~K}$ for $x=0.05$ to $177 \mathrm{~K}$ for $x=0.30$.

It is worth to note that all the samples exhibit another transition from insulator to metal at $T_{\min }$ well below $100 \mathrm{~K}$ with increase in temperature, known as resistivity minimum behaviour, observed in all the samples. It is known that the resistivity in the metallic region is due to the various electron-phonon and electron-magnon inelastic scattering processes. The appearance of an insulating/semiconducting behaviour in the low temperature range (below $\sim 50 \mathrm{~K}$ ) is an anomaly and reproduced by adding e-e elastic scattering term to inelastic scattering term as follows: $\rho=\rho_{\text {elastic }}+$ $\rho_{\text {inelastic }}$, where $\rho_{\text {elastic }}$ is the correction to resistivity due to low temperature elastic e-e scattering and $\rho_{\text {inelastic }}$ is the resistivity contribution from electron-phonon and electronmagnon inelastic scattering processes. $\rho_{\text {inelastic increases as }}$ temperature increases, whereas $\rho_{\text {elastic }}$ is dominant at low temperatures. Low temperature correction to the resistivity is given by $\rho_{\text {elastic }}$ which is quantified as follows: $\rho_{\text {elastic }}=1 /\left(\sigma_{0}+B T^{1 / 2}\right)$, where $\sigma_{0}$ is the residual conductivity contributed by the temperature-independent scattering processes and $B$ is proportional to the diffusion constant that depicts the depth of the resistivity minimum. However, inelastic scattering, which increases monotonically with temperature, compete with the e-e interaction (which decreases with the temperature as described in the equation of $\rho_{\text {inelastic }}$ ) and gives rise to the minimum resistivity. Assuming all the temperature-dependent scattering processes, like electron-phonon, electron-magnon and electron-electron, which are adequately described by the single power law, inelastic resistivity also can be described as follows: $\rho_{\text {elastic }}=$ $\rho_{n} T^{n}$. In this equation, $\rho_{n}$ is the coefficient of $T^{n}$ that

Table 1. Values of Rietveld refined lattice parameters and cell volume for $\mathrm{La}_{0.5} \mathrm{Pr}_{0.2} \mathrm{Ca}_{0.3-x} \mathrm{Ba}_{x} \mathrm{MnO}_{3}$ (LPCBMO) $(0.05 \leq x \leq 0.30)$ manganites.

\begin{tabular}{lcccc}
\hline Sample & $a(\AA)$ & $b(\AA)$ & $c(\AA)$ & $V\left(\AA^{3}\right)$ \\
\hline $\mathrm{La}_{0.5} \mathrm{Pr}_{0.2} \mathrm{Ca}_{0.25} \mathrm{Ba}_{0.05} \mathrm{MnO}_{3}$ & $5.451(3)$ & $7.752(6)$ & $5.512(2)$ & 232.9159 \\
$\mathrm{La}_{0.5} \mathrm{Pr}_{0.2} \mathrm{Ca}_{0.2} \mathrm{Ba}_{0.1} \mathrm{MnO}_{3}$ & $5.472(6)$ & $7.768(1)$ & $5.521(6)$ & 234.6784 \\
$\mathrm{La}_{0.5} \mathrm{Pr}_{0.2} \mathrm{Ca}_{0.15} \mathrm{Ba}_{0.15} \mathrm{MnO}_{3}$ & $5.489(1)$ & $7.781(2)$ & $5.534(7)$ & 236.3566 \\
$\mathrm{La}_{0.5} \mathrm{Pr}_{0.2} \mathrm{Ca}_{0.05} \mathrm{Ba}_{0.25} \mathrm{MnO}_{3}$ & $5.509(5)$ & $7.789(3)$ & $5.541(5)$ & 237.7621 \\
$\mathrm{La}_{0.5} \mathrm{Pr}_{0.2} \mathrm{Ba}_{0.3} \mathrm{MnO}_{3}$ & $5.512(2)$ & $7.791(2)$ & $5.548(2)$ & 238.2533 \\
\hline
\end{tabular}



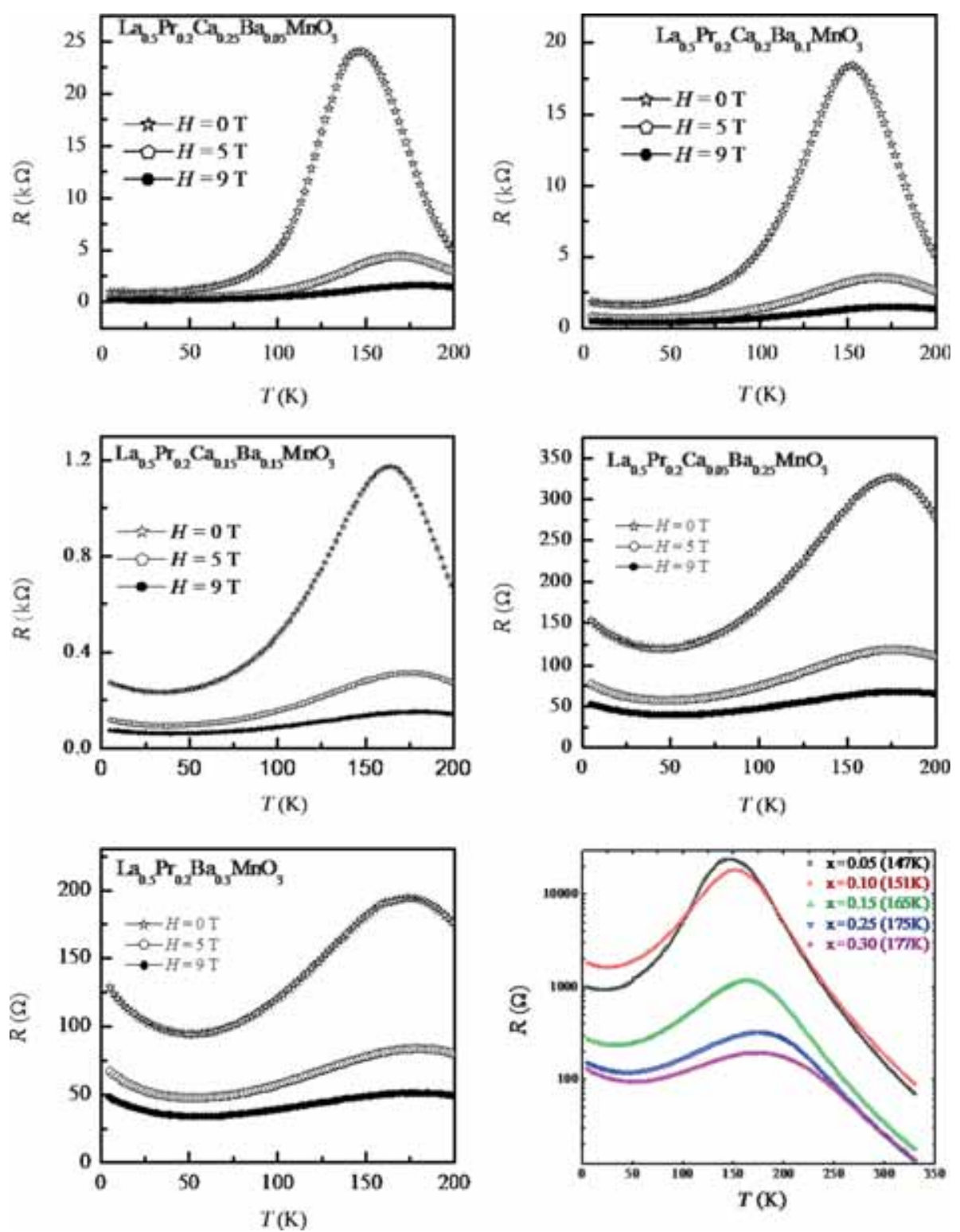

Figure 3. (Colour online) Variation in resistance with temperature in the range 5-330 $\mathrm{K}$ under 0,5 and $9 \mathrm{~T}$ fields for $\mathrm{La}_{0.5} \mathrm{Pr}_{0.2} \mathrm{Ca}_{0.3-x} \mathrm{Ba}_{x} \mathrm{MnO}_{3}$ (LPCBMO) $(0.05 \leq x \leq 0.30)$ manganites. Last plot shows the zero field resistance variation with temperature for all the samples. Values given in the brackets show the metal-insulator transition temperatures $\left(T_{\mathrm{P}}\right)$.

depends on the contribution of the inelastic scattering. Now, the total resistivity can be expressed as follows: $\rho=\rho_{\text {elastic }}+$ $\rho_{\text {inelastic }}=\left[1 /\left(\sigma_{0}+B T^{1 / 2}\right)\right]+\rho_{n} T^{n}$. Values of exponent $n$ indicate the nature of the electron scattering with magnon, since magnon is the magnetic lattice vibrations and its interactions with the electrons resulting in the electron-magnon scattering processes. Expression for the total resistivity $[\rho=$ $\left.\left\{1 /\left(\sigma_{0}+B T^{1 / 2}\right)\right\}+\rho_{n} T^{n}\right]$ has been fitted to the experimental results to understand the role of magnetic field in modifying the nature of the magnon and its interactions with the charge carriers (electrons).

To justify the electron-electron scattering/interaction, as a possible cause for the resistivity minimum behaviour observed for the present case of LPCBMO system, the low temperature resistivity data were fitted for all the LPCBMO samples under 0,5 and $9 \mathrm{~T}$ fields to $\rho=\left[1 /\left(\sigma_{0}+B T^{1 / 2}\right)\right]$ $+\rho_{n} T^{n}$ equation. All the fitting parameters, obtained from the electron-electron scattering model fittings are tabulated in table 2. From table 2, one can state that $\chi^{2}$ is below 1 , accept for $x=0.10$ sample, in which it is little higher, which shows the good quality of the fittings. $\chi^{2}$ decreases sharply with the increase in size disorder (from $x=0.05$ 0.30 ) which suggests that low temperature minima becomes more effective with the increase in Ba-content and with the increase in size disorder, it fits our theoretical model very perfectly. From figure 4 , it is clearly seen that the value of $T_{\min }$ increases as Ba-content/size disorder increases, simultaneously residual resistivity also increases with the 
Table 2. Values of parameters obtained from fitting the low-temperature resistivity data to the equation $\rho=\left[1 /\left(\sigma_{0}+B T^{1 / 2}\right)\right]+\rho_{n} T^{n}$ under 0, 5 and $9 \mathrm{~T}$ applied fields for $\mathrm{La}_{0.5} \mathrm{Pr}_{0.2} \mathrm{Ca}_{0.3-x} \mathrm{Ba}_{x} \mathrm{MnO}_{3}$ (LPCBMO) $(0.05 \leq x \leq 0.30)$ manganites.

\begin{tabular}{|c|c|c|c|c|c|c|c|c|}
\hline Sample & $T_{\min }(\mathrm{K})$ & $H(\mathrm{~T})$ & $\sigma_{0}\left(\Omega^{-1} \mathrm{~cm}^{-1}\right)$ & $B\left(\Omega^{0.5} \mathrm{~cm}^{0.5}\right)$ & $\rho_{n}\left(\Omega \mathrm{cm} / \mathrm{K}^{n}\right)$ & $n$ & $\chi^{2}$ & $R^{2}$ \\
\hline \multirow[t]{3}{*}{$x=0.05$} & 18 & 0 & 0.00092 & 0.00004 & 0.00135 & 3.14 & 6.21000 & 0.99814 \\
\hline & 25 & 5 & 0.00179 & 0.00023 & 0.00657 & 2.51 & 5.31335 & 0.98892 \\
\hline & 27 & 9 & 0.00382 & 0.00026 & 0.00093 & 2.78 & 0.14636 & 0.99786 \\
\hline \multirow[t]{3}{*}{$x=0.10$} & 27 & 0 & 0.00136 & 0.00008 & 0.00044 & 3.26 & 1.72500 & 0.99766 \\
\hline & 30 & 5 & 0.00260 & 0.00037 & 0.00120 & 2.75 & 0.63956 & 0.99790 \\
\hline & 35 & 9 & 0.00515 & 0.00046 & 0.00012 & 3.11 & 0.13600 & 0.99788 \\
\hline \multirow[t]{3}{*}{$x=0.15$} & 33 & 0 & 0.00876 & 0.00064 & 0.00002 & 3.38 & 0.03882 & 0.99795 \\
\hline & 37 & 5 & 0.01763 & 0.00213 & $9.86 \mathrm{E}-6$ & 3.39 & 0.00014 & 0.99839 \\
\hline & 40 & 9 & 0.03048 & 0.00266 & $2.54 \mathrm{E}-7$ & 4.08 & 0.00720 & 0.99757 \\
\hline \multirow[t]{3}{*}{$x=0.25$} & 46 & 0 & 0.01409 & 0.00127 & $2.22 \mathrm{E}-8$ & 4.70 & 0.00841 & 0.99952 \\
\hline & 50 & 5 & 0.02632 & 0.00312 & $4.85 \mathrm{E}-9$ & 5.00 & 0.00624 & 0.99903 \\
\hline & 57 & 9 & 0.03959 & 0.00421 & $3.80 \mathrm{E}-10$ & 5.50 & 0.00330 & 0.99878 \\
\hline \multirow{3}{*}{$x=0.30$} & 57 & 0 & 0.01735 & 0.00191 & $9.30 \mathrm{E}-10$ & 5.40 & 0.00698 & 0.99950 \\
\hline & 59 & 5 & 0.03156 & 0.00427 & $4.31 \mathrm{E}-10$ & 5.47 & 0.00866 & 0.99836 \\
\hline & 65 & 9 & 0.04522 & 0.00572 & $2.37 \mathrm{E}-14$ & 7.80 & 0.00514 & 0.99804 \\
\hline
\end{tabular}

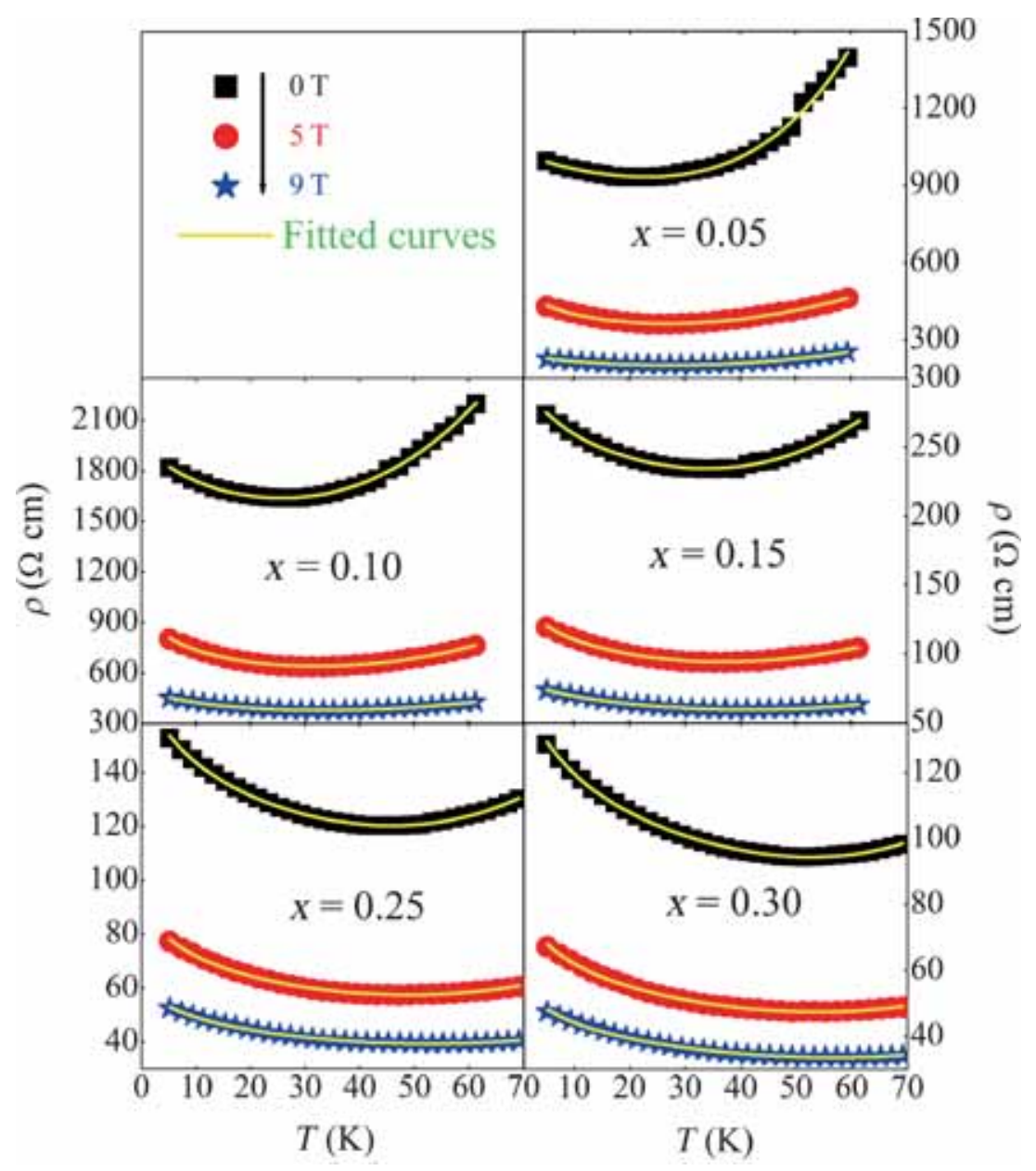

Figure 4. (Colour online) Fitting of low-temperature resistivity data to electron-electron interaction of the form $\rho=\left[1 /\left(\sigma_{0}+B T^{1 / 2}\right)\right]+\rho_{n} T^{n}$, under the fields of 0,5 and $9 \mathrm{~T}$ for $\mathrm{La}_{0.5} \operatorname{Pr}_{0.2} \mathrm{Ca}_{0.3-x} \mathrm{Ba}_{x} \mathrm{MnO}_{3}$ (LPCBMO) $(0.05 \leq x \leq 0.30)$ manganites. 
size disorder which is shown in table 2. Hence, from table 2, it can be seen that as size disorder increases, role of elastic scattering becomes dominant. This may be understood in terms of electron localization: as size disorder increases, the angle between $\mathrm{Mn}-\mathrm{O}-\mathrm{Mn}$ bond angles gets modified. At low temperature, in the absence of thermal activation energy, spins are localized at Mn orbits and resistivity shows upturn in its nature. With applied field, upturn shifts towards higher temperature along with the increase in residual conductivity, inverse of depth of minima (parameter $B$ ) and parameter $n$.

Uncooled infrared imaging systems based on microbolometers offer the possibility for the development of a light weight, inexpensive alternative for complex, cooled IR imaging systems based on the $\mathrm{HgCdTe}$ (MCT) or InSb. The sensitivity of presently used micro-bolometer-based systems is one order of magnitude lower than that of semiconducting systems. The active element in the micro-bolometer based detector is an oxide-coated silicon micro-bridge. The oxide film has a temperature-dependent resistance. Heating of the micro-bridge by the incident IR radiation is detected by a change in the micro-bridge resistivity [27-29].

Due to the advances in thermoelectric cooling, materials with high thermal nonlinearities in the temperature range of 250-300 K are potential candidates for bolometric sensors. The figure of merit for bolometric materials is the thermal coefficient of resistance (TCR) and the noise volume (S) [29]. The commercial bolometers based on $\mathrm{VO}_{x}$ use TCR values around $2.5-4.0 \%$. In comparison, TCR values ranging from 18 to $20 \%$ are possible in the manganites over the very same temperature range [7]. The TCR is very closely related to the magnetic homogeneity of the material and as a matter of fact that there is a strong correlation between TCR and the thermal coefficient of the magnetization (TCM), the inverse ferromagnetic resonance (FMR) line width and the value of the total magnetization. The TCR value drops with the increase in the Curie temperature, which is an indication of an intrinsic thermal inhomogeneity in these manganites.
Early noise measurements in the manganites also showed very large noise volumes in those materials which were typically four to six orders of magnitude larger than that was seen in other metal oxide conductors such as YBCO. However, in recent times, significantly reduced noise volumes in higher quality manganite films was observed and there was a clear correlation with increased TCR values. Noise characteristics of CMR already reported in comparison with other oxide conductors such as YBCO. The films made today with much higher TCR values also show two orders of magnitude reduction in the noise volumes. The discovery of new materials with higher TCR would increase the sensitivity of the micro-bolometers comparable with MCT detectors [29].

The value of TCR is very useful for device application, which can be calculated as follows: TCR $=(1 / R)(\mathrm{d} R / \mathrm{d} T)$ $\times 100\left(\% \mathrm{~K}^{-1}\right)$. The LPBMO sample at higher temperature $\sim 1350^{\circ} \mathrm{C}$ for $24 \mathrm{~h}$ in air atmosphere was sintered and the TCR was calculated by performing $R-T$ measurements under zero applied field. Figure 5 shows the variation in TCR with temperature under zero applied field for LPBMO ( $x=0.30)$ manganites sintered at (a) $1300^{\circ} \mathrm{C}$ and (b) $1350^{\circ} \mathrm{C}$. It is clearly seen that with increase in sintering temperature under the same environment and for the same time duration, TCR gets enhanced for almost three times as compared to lower-sintered LPBMO sample understudy. It is well-established fact that with increase in sintering temperature, grain size increases, while grain boundary density decreases [30-33]. To confirm the microstructural changes for the LPBMO samples sintered at different temperatures, SEM measurements for both these samples were performed as shown in the inset of figure 5. The grain size increases while the grain boundaries become sharp at higher sintered sample (figure 5a). This also affects the sharpness of the TCR peak in figure 5a. This can also be attributed to the large grain size and sharp grain boundaries in higher sintered sample. These observations suggest that microstructural changes can affect efficiently the
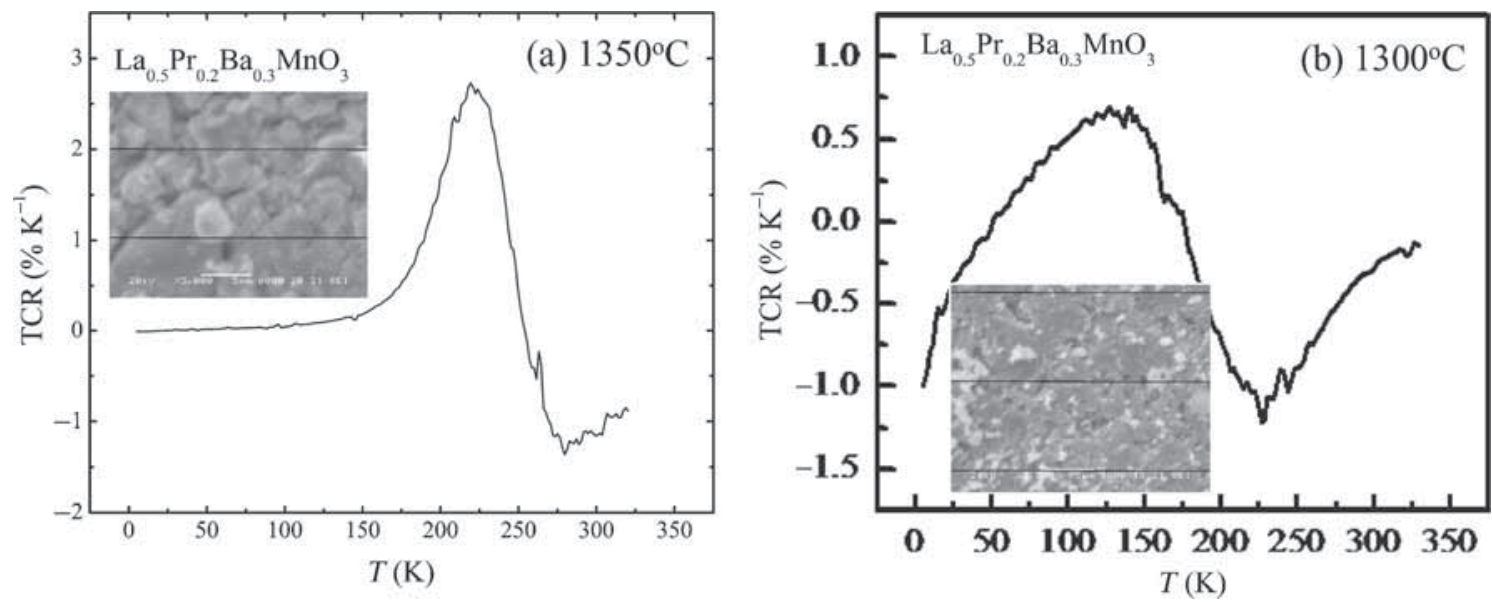

Figure 5. Variation in TCR with temperature under zero applied field for $\mathrm{La}_{0.5} \operatorname{Pr}_{0.2} \mathrm{Ba}_{0.3} \mathrm{MnO}_{3}(\mathrm{LPBMO})(x=0.30)$ manganites sintered at different temperatures. 

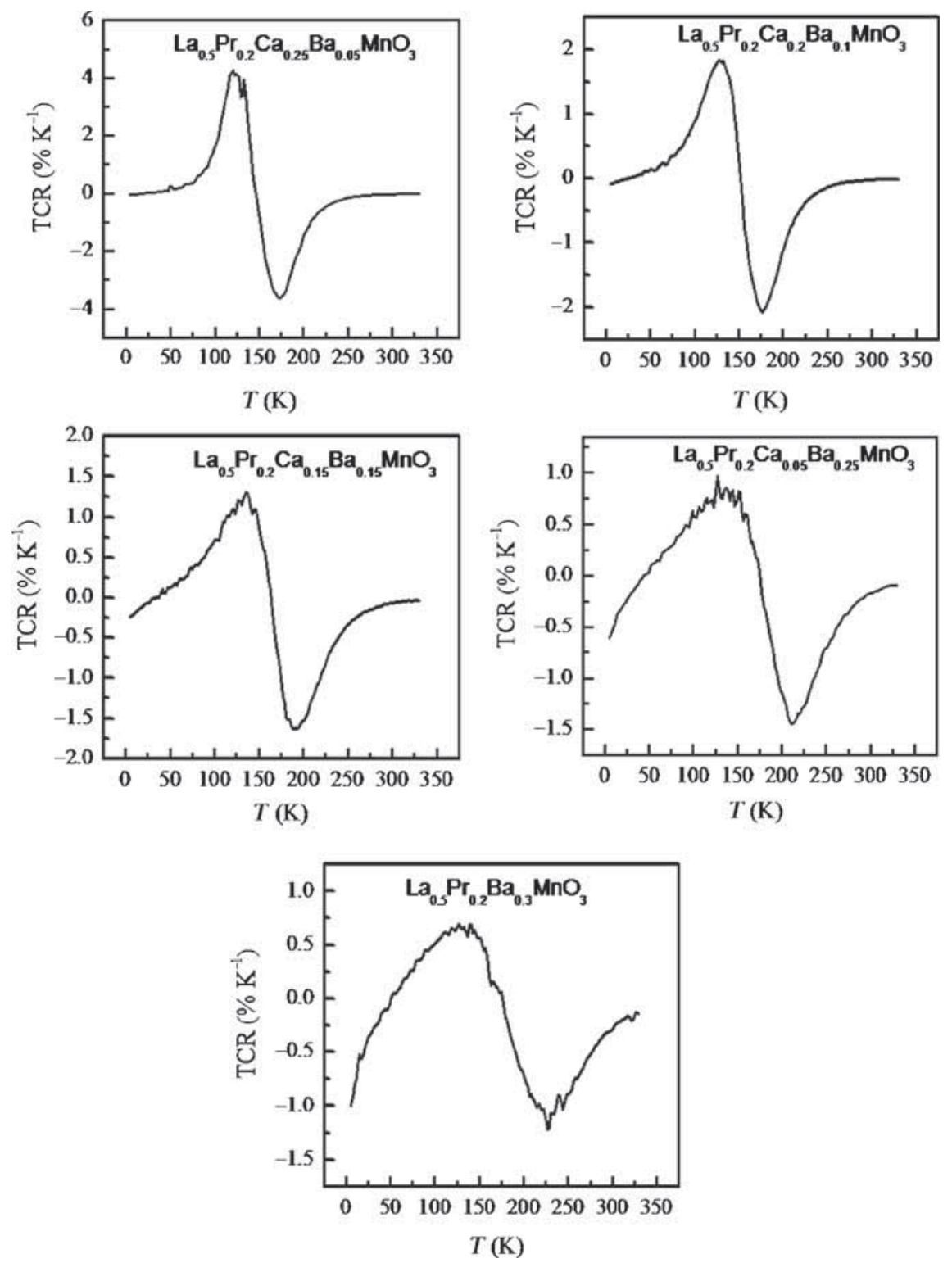

Figure 6. Variation in TCR with temperature under zero applied field for $\mathrm{La}_{0.5} \mathrm{Pr}_{0.2} \mathrm{Ca}_{0.3-x} \mathrm{Ba}_{x} \mathrm{MnO}_{3}$ (LPCBMO) $(0.05 \leq x \leq 0.30)$ manganites.

Table 3. Values of positive and negative TCR under zero applied field for $\mathrm{La}_{0.5} \mathrm{Pr}_{0.2} \mathrm{Ca}_{0.3-x} \mathrm{Ba}_{x} \mathrm{MnO}_{3}$ (LPCBMO) $(0.05 \leq x \leq 0.30)$ manganites.

\begin{tabular}{lcc}
\hline Sample & Maximum positive TCR & Maximum negative TCR \\
\hline $\mathrm{La}_{0.5} \mathrm{Pr}_{0.2} \mathrm{Ca}_{0.25} \mathrm{Ba}_{0.05} \mathrm{MnO}_{3}$ & $4.09 \% \mathrm{~K}^{-1}(122 \mathrm{~K})$ & $-3.65 \% \mathrm{~K}^{-1}(169 \mathrm{~K})$ \\
$\mathrm{La}_{0.5} \mathrm{Pr}_{0.2} \mathrm{Ca}_{0.2} \mathrm{Ba}_{0.1} \mathrm{MnO}_{3}$ & $1.79 \% \mathrm{~K}^{-1}(129 \mathrm{~K})$ & $-2.06 \% \mathrm{~K}^{-1}(176 \mathrm{~K})$ \\
$\mathrm{La}_{0.5} \mathrm{Pr}_{0.2} \mathrm{Ca}_{0.15} \mathrm{Ba}_{0.15} \mathrm{MnO}_{3}$ & $1.28 \% \mathrm{~K}^{-1}(135 \mathrm{~K})$ & $-1.61 \% \mathrm{~K}^{-1}(190 \mathrm{~K})$ \\
$\mathrm{La}_{0.5} \mathrm{Pr}_{0.2} \mathrm{Ca}_{0.05} \mathrm{Ba}_{0.25} \mathrm{MnO}_{3}$ & $0.82 \% \mathrm{~K}^{-1}(115 \mathrm{~K})$ & $-1.42 \% \mathrm{~K}^{-1}(212 \mathrm{~K})$ \\
$\mathrm{La}_{0.5} \mathrm{Pr}_{0.2} \mathrm{Ba}_{0.3} \mathrm{MnO}_{3}$ & $0.60 \% \mathrm{~K}^{-1}(115 \mathrm{~K})$ & $-1.16 \% \mathrm{~K}^{-1}(228 \mathrm{~K})$ \\
\hline
\end{tabular}

temperature sensitivity for manganite compounds. Detailed discussion in the present study on LPCBMO samples is as follows.
Figure 6 shows the variation in TCR with temperature under zero applied field for all the LPCBMO samples understudy. Values of positive and negative TCR for all the 
samples studied are listed in table 3. It can be seen from figure 6 and table 3 that with the increase in Ba-content and hence, size disorder, the capability of the samples to sense the change in temperature, i.e., temperature sensitivity, decreases from $4.09 \% \mathrm{~K}^{-1}(x=0.05)$ to $0.6 \% \mathrm{~K}^{-1}(x=0.30)$ (positive TCR $)$ and $-3.65 \% \mathrm{~K}^{-1}(x=0.05)$ to $-1.16 \% \mathrm{~K}^{-1}$ $(x=0.30)$ (negative TCR). Also, one noticeable observation is that the sharpness in the TCR curve is higher in lowerdoped samples, while with increase in the doping of $\mathrm{Ba}$ (size disorder is increasing), nature of TCR curve becomes more broader. In the last sample of this series, LPBMO $(x=0.30)$, exhibits very large broadening effect in the TCR curve. This behaviour also observed in the $R-T$ plots of LPCBMO $(0.05$ $\leq x \leq 0.3)$ samples. This may be understood in terms of the phase separation and grain boundary effect is explained below.

This anomaly is not in accordance with the ZDE interactions and can be explained in the purview of possible grain boundary localization or phase separation. It is important to mention that the polycrystalline samples possess various micron-sized grains, in which properties may vary inside the grain and on the grain boundaries. As the ZDE comes into the play, the bulk part of the grain becomes ferromagnetic metallic, while the grain boundaries are still insulating. The grain boundaries may be insulating because of the grain boundary pinning of Mn ion spins, poor connectivity and grain boundary contamination. This creates the insulating channels at the grain boundaries and does not allow the percolation of the charge carriers and results in insulating behaviour even after the onset of $T_{\mathrm{C}}$. Once the grain boundaries become conducting at lower temperatures, the insulator-metal $(I-M)$ transitions appear. Other factor that can cause a disparity between the sharper TCR curve in lower size-disordered samples and broader TCR curve in large size-disordered samples is the phase separation. Due to the granular mixture of different electronic densities, the $T_{\mathrm{C}}$ of different grains could be different. For instance, we assume that majority of the grains have large electronic density so that they exhibit $T_{\mathrm{C}}$ at higher temperatures, while the remaining minor fraction have lower $T_{\mathrm{C}}$. Now, at the onset of the $T_{\mathrm{C}}$ of majority of grains (with higher $T_{\mathrm{C}}$ ), the system tends to be ferromagnetic even as a minority of grain are still paramagnetic insulating. In such a situation, though the system is a ferromagnetic, it will continue to exhibit semiconducting/insulating behaviour unless all the grains become ferromagnetic. At a certain temperature when the whole system attains ferromagnetism, percolation is possible and the system exhibits $I-M$ transition.

\section{Conclusions}

In summary, high-quality bulk samples of LPCBMO (0.05 $\leq x \leq 0.30)$ manganites were synthesized using conventional solid-state reaction. The suppression in resistivity and increase in $T_{\mathrm{P}}$ with size disorder/Ba-content have been understood in terms of improved one electron bandwidth in the system. Strong resistivity minimum behaviour and its modifications due to Ba-content and applied magnetic field have been discussed in detail in the light of electron-electron scattering mechanism at low temperature, where large coulombic interactions are expected. Size disorder-induced modifications in the temperature sensitivity have been discussed in the context of possible phase separation scenario and grain boundary effect.

\section{Acknowledgement}

ZJ is thankful to UGC, New Delhi, for financial support in the form of UGC (BSR) meritorious fellowship (file no. F.25-1/2013-14 (BSR)/7-156/2007(BSR)).

\section{References}

[1] Blasco J, Garcia J, De Teresa J M, Ibarra M R, Perez J, Algarabel P A et al 1997 Phys. Rev. B 558905

[2] Chahara K, Ohno T, Kasai M and Kozono Y 1993 Appl. Phys. Lett. 631990

[3] Dagotto E, Hotta T and Moreo A 2001 Phys. Rep. 3441

[4] Khachar Uma, Solanki P S, Choudhary R J, Phase D M, Ganesan V and Kuberkar D G 2012 Solid State Commun. 152 34

[5] Khachar U D, Solanki P S, Choudhary R J, Phase D M, Ganesan V and Kuberkar D G 2012 Appl. Phys. A 108733

[6] Kataria Bharat, Solanki P S, Khachar Uma, Vagadia Megha, Ravalia Ashish, Keshvani M J et al 2013 Radiat. Phys. Chem. 85173

[7] Khachar Uma, Solanki P S, Choudhary R J, Phase D M and Kuberkar D G 2013 J. Mater. Sci. Technol. 29989

[8] Khachar Uma, Solanki P S, Kansara Sanjay, Choudhary R J, Phase D M, Kuberkar D G et al 2013 IEEE Trans. Nanotechnol. 12915

[9] Zener C 1951 Phys. Rev. 81440

[10] Zener C 1951 Phys. Rev. 82403

[11] Millis A J, Littlewood P B and Shraiman B I 1995 Phys. Rev. Lett. 745144

[12] Millis A J, Shraiman B I and Mueller R 1996 Phys. Rev. Lett. 77175

[13] Ramirez A P 1997 J. Phys.: Condens. Matter 98171

[14] Hwang H Y, Cheong S W, Radaelli P G, Marezio M and Batlogg B 1995 Phys. Rev. Lett. 75914

[15] Rodriguez-Martinez L M and Attfield J P 1996 Phys. Rev. B 54 R 15622

[16] Rana D S, Mavani K R, Thaker C M, Kuberkar D G, Kundaliya D C and Malik S K 2004 J. Appl. Phys. 957097

[17] Solanki P S, Doshi R R, Ravalia Ashish, Keshvani M J, Pandya Swati, Ganesan V et al 2015 Physica B 46571

[18] Rathod J S, Khachar Uma, Doshi R R, Solanki P S and Kuberkar D G 2012 Int. J. Mod. Phys. B 261250136

[19] Rana D S, Markna J H, Parmar R N and Kuberkar D G 2005 Phys. Rev. B 71212404

[20] Solanki P S, Doshi R R, Khachar U D, Choudhary R J and Kuberkar D G 2011 Mater. Res. Bull. 461118 
[21] Schiffer P, Ramirez A P, Bao W and Cheong S W 1995 Phys. Rev. Lett. 753336

[22] Tokura Y and Tomioka Y 1999 J. Magn. Magn. Mater. 2001

[23] Sharma N, Nigam A K, Pinto R, Venkataramani N, Prasad S, Chandra G et al 1995 J. Magn. Magn. Mater. 166 65

[24] De Gennes P G 1960 Phys. Rev. 118141

[25] Thaker C M, Rana D S, Mavani Krushna, Patil S I, Sahasrabudhe M and Kuberkar D G 2003 Ind. J. Eng. Mater. Sci. 10324

[26] Rana D S, Thaker C M, Mavani Krushna, Rayaprol S and Kuberkar D G 2002 Proc. DAE SSPS 45489

[27] Rajeswari M, Chen C H, Goyal A, Kwo C, Robson M C, Ramesh R et al 1996 Appl. Phys. Lett. 683555
[28] Ogale S B, Shreekala R, Bathe R, Date S K, Patil S I, Hannoyer B et al 1998 Phys. Rev. B 577841

[29] Kumar Ravi, Choudhary R J, Patil S I, Hussain Shahid, Srivastava J P and Malik S K 2005 Appl. Phys. Lett. 86 222501

[30] Solanki P S, Doshi R R, Thaker C M, Pandya Swati, Ganesan V and Kuberkar D G 2009 J. Nanosci. Nanotechnol. 95681

[31] Solanki P S, Doshi R R, Khachar U D, Vagadia M V, Ravalia A B, Kuberkar D G et al 2010 J. Mater. Res. 251799

[32] Kuberkar D G, Doshi R R, Solanki P S, Khachar Uma, Vagadia Megha, Ravalia Ashish et al 2012 Appl. Surf. Sci. 258 9041

[33] Kansara S B, Dhruv Davit, Joshi Zalak, Pandya D D, Rayaprol S, Solanki P S et al 2015 Appl. Surf. Sci. 3561272 